\title{
SYMPOSIUM
}

\section{STI epidemics in the Indian Ocean region: can the phase be assessed?}

\author{
A Matteelli, C Pizzocolo, H Abdou Bakar, M Toyb, N Pyndiah, N E Udonwa, \\ A G Gedeon, C Farina, S Caligaris, F Castelli, G Carosi
}

The Indian Ocean provides a unique opportunity to curb the HIV epidemic in its nascent phase through strengthening STI control programmes. Making effective and appropriate health services available should be regarded as the first priority for STI control in the region and, whenever possible, core groups should be identified and targeted to interrupt transmission within such networks.

See end of article for authors' affiliations

Correspondence to Dr Alberto Matteelli, Clinic of Infectious and Tropical Diseases, Piazza Spedali Civili 1, 25123 Brescia, Italy;

forleo@master.cci.unibs.it

Accepted

30 November 2001 n the attempt to deepen our understanding of the transmission dynamics of sexually transmitted diseases, Wasserheit and Aral have postulated a model in which the subpopulation structure of each individual sexually transmitted infection (STI) epidemic evolves through phases, driven by a reciprocal interaction with preventive interventions. ${ }^{1}$ The model shows consistency with the findings of epidemiological studies in the USA and Canada. ${ }^{2}$ The importance of the proposed concept model lies in its implications for the development of phase specific STI prevention strategies and research. It is therefore particularly relevant to look for epidemiological evidence supporting the validity of the model in the setting of resource-poor countries, in which most of the worldwide burden of STI is concentrated..$^{3}$ In this paper, we will discuss this hypothesis in the light of the current STI situation in the Comoros, Mauritius, and Seychelles in the Indian Ocean region. Some of the epidemiological data presented have been generated in the context of an intervention for the control of STI and AIDS conducted with the support of the European Commission from 1996 to 1998.

\section{THE GEOGRAPHICAL CONTEXT}

The Comoros, Mauritius, and Seychelles are small island countries which lie in the same geographical region but are heterogeneous in terms of socioeconomic conditions and health care system structure. lation of 76000 and an annual growth rate of $1 \%$. In the second half of the 1990s, $31 \%$ of the population were under 15 years of age, mean life expectancy was 65 years for men and 71 for women, infant mortality was below 7/1000 live births, and immunisation coverage against measles was about $100 \%$.

Mauritius has a larger population of 1141508 , but similar vital statistics: growth rate of $1 \%, 27 \%$ of the population under 15 years, mean life
Seychelles is the smallest country, with a popu- expectancy of 68 years for men and 75 for women. Infant mortality rate has been reduced from $38 / 1000$ in 1978 to $16 / 1000$ in 1998 .

The total population of the Comoros is estimated to be 517 29l. Vital statistics are more like those of neighbouring sub-Saharan African countries, with an annual growth of $3.1 \%, 45 \%$ of the population under 15 years of age, and mean life expectancy of 55 years for men and 57 for women. The infant mortality rate is $76 / 1000$, among the highest in Africa.

A gradient can be identified in the economic indicators of the three countries. Seychelles is relatively rich, with a gross national product (GNP) per capita which rose from US\$2260 to US\$3452, reaching US\$6910 in 1998. In Mauritius the GNP per capita increased from US\$1480 in 1990 to US\$3710 in 1999. The Comoros are economically disadvantaged: in 1998 the GNP per capita was US\$450, decreasing by $2.1 \%$ in the period 1986 to 1992.

\section{PUBLIC HEALTH ORGANISATION AND STI PREVENTION PROGRAMMES}

The Seychelles benefits from a relatively high standard of public health care and since 1979 the medical services have been available without cost to virtually every inhabitant. In Mauritius public health services are also free and easily accessible, though the population per doctor is more than 10 times higher in the Seychelles (one doctor per 1400 inhabitants) than in Mauritius (one doctor per 14323 inhabitants). Since 1995 the Comoros have adopted a decentralised system of primary health care; although health care is still publicly financed, individual health units are relatively autonomous and financially independent: clients are charged for any health service in order to create a sustainable public health system. ${ }^{5}$ Private medical practice is uncommon in the Seychelles and virtually unknown in the Comoros. On the other hand, it is very common in Mauritius, where it is thought to account for more than 50\% of all STI care practices.

STI control programmes operate in each of the three countries. These advocate strategies to reduce the rate of sex partner change through an education campaign, and to reduce the efficiency of transmission through condom promotion. In addition the strengthening of case management for STI patient was the object of a specific regional project in the second half of the 1990s.

STI care in the Seychelles is concentrated in one categorical clinic in the capital, where high quality comprehensive activities are carried out, 

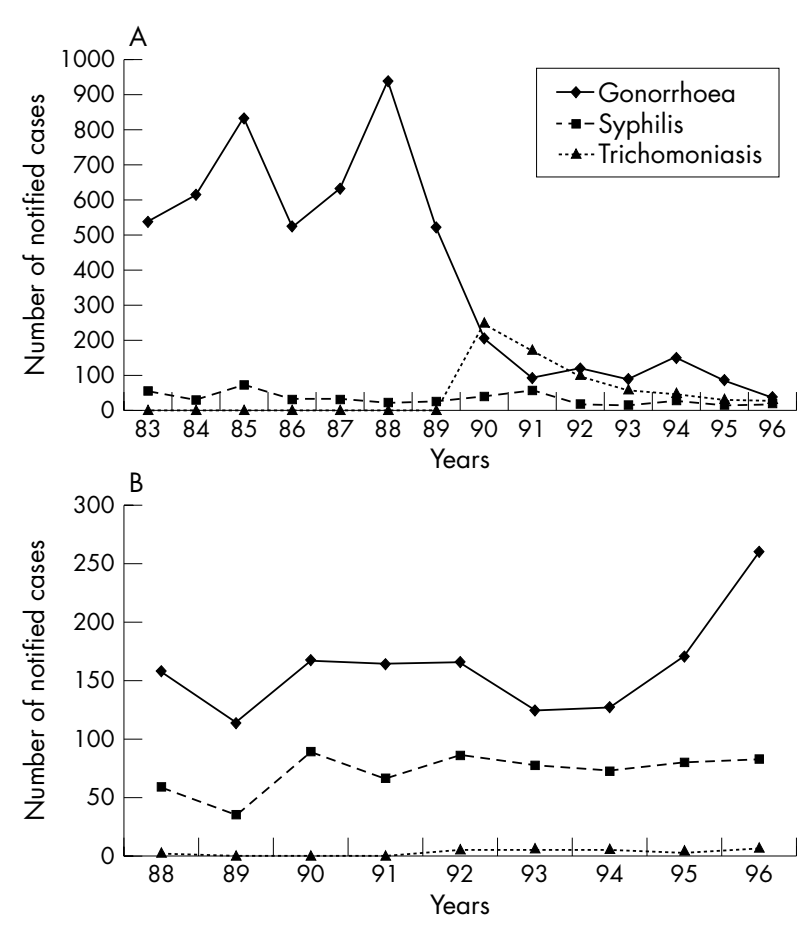

Figure 1 Notifiable sexually transmitted infections in the Republic of Seychelles (A); Mauritius (B).

including partner notification. Such a centre based, semivertical approach to STI case management seems to be appropriate for this small country.

A similar vertical and centralised system has been adopted in Mauritius: one categorical STI clinic situated in the capital town is committed to managing patients from all over the country. However, because a very small fraction of STI patients is thought to attend the clinic, a process of decentralisation and integration of STI services into the public health care (PHC) system has been launched in 1998.

In the Comoros no specialised STI centre is available. In 1998 the Government of the Comoros adopted a policy for the decentralisation and integration of STI care services into the PHC system using the syndromic approach, but the quality of STI services at PHC is currently believed to be rather poor.

In summary, in the past, STI care practices of acceptable quality have been widely available in the Seychelles, available on limited scale in Mauritius, and virtually unavailable in the Comoros.

\section{STI DESCRIPTIVE EPIDEMIOLOGY}

The two categorical STI clinics in Mauritius and the Seychelles provide case notifications for STIs, based on a mixture of microbiological (gonorrhoea in men, trichomoniasis, and so on) and clinical criteria (non-gonococcal urethritis, genital herpes, chancroid). The trends of selected reported STIs over a
10 year period are reported in fig $1 \mathrm{~A}$ and B. Apparently, all bacterial STI are decreasing in the Seychelles, while they are increasing or stable in Mauritius, but the interpretation of these figures is difficult. Variations in notified cases may represent internal inconsistency in diagnosis and/or reporting rather than true variations in incidence rates.

Syphilis life time infection rates (either positive VDRL, positive TPHA, or both positive) have been monitored in the three countries, though measurements are jeopardised over time and space. In the Comoros, prevalence ranged between 3.5\% and 6.9\% among pregnant women during 1995 to 1997. In Mauritius in 1998 syphilis rates were $0.4 \%$ among pregnant women, $1.2 \%$ among blood donors, and 30\% among STI patients. In the Seychelles, TPHA seropositivity ranges in 1993/1994 were $1.0-2.2 \%$ among pregnant women, $1.2-1.4 \%$ among blood donors, and 9.4-19.3\% among STI patients. These data show the high seroprevalence of syphilis in the general population in the Comoros, while in Mauritius and the Seychelles high rates are present in selected populations only.

Limited information on point prevalence of selected bacterial STIs has been obtained in the period 1996 to 1998 through special surveys on small samples of low and high risk female populations (table 1). Results of measurements among pregnant women reflect those of the general population. In agreement with the data on syphilis surveillance, prevalences of gonorrhoea, syphilis, and trichomoniasis were very high in the Comoros and significantly lower in Mauritius and the Seychelles. ${ }^{6}$ In contrast to what is observed in the general population, the rates of bacterial STI among commercial sex workers were relatively higher in Mauritius than in the Comoros (table 1). Data on symptomatic women are more difficult to interpret. The women sampled in these surveys should be regarded as moderately low risk populations, as women presenting with vaginal discharge had a diagnosis of cervicitis in only $14 \%$ and $8 \%$ in the Comoros and Seychelles, respectively, the most frequent aetiological diagnosis being vaginal candidiasis. ${ }^{7-9}$ Finally, in a sample of 81 men attending the same STI clinic with urethral discharge in the Seychelles, $26 \%$ had chlamydia infection and 38\% gonococcal infection ( $11 \%$ mixed infections), showing that both these bacterial STI are still responsible for the majority of cases of symptomatic urethritis in men. ${ }^{10}$

A study of minimum inhibitory concentrations (MIC) on N gonorrhoeae isolates from 67 strains isolated at Candos Hospital, Mauritius in 1997, using either the E test or the standard broth dilution methods, showed high resistance levels to penicillin $\left(\mathrm{MIC}_{50}, 16 \mu \mathrm{g} / \mathrm{ml}\right)$, tetracyclines $\left(\mathrm{MIC}_{50}, 4 \mu \mathrm{g} / \mathrm{ml}\right)$, and cotrimoxazole $\left(\mathrm{MIC}_{50}, 4 \mathrm{\mu g} / \mathrm{ml}\right.$ ), and full sensitivity to ceftriaxone $\left(\mathrm{MIC}_{95}, 0.047 \mathrm{\mu g} / \mathrm{ml}\right)$ and ciprofloxacine $\left(\mathrm{MIC}_{95}, 0.47\right.$ $\mu \mathrm{g} / \mathrm{ml}$ ) (fig 2).

\section{HIV EPIDEMIOLOGY}

Since 1987 the National AIDS control programmes of the three countries have provided data from laboratory HIV tests and HIV infection/AIDS notifications. Between 1987 and 1997 the cumulative number of cases of HIV infection/AIDS was 41 in the Comoros, 168 in Mauritius, and 79 in the

Table 1 Per cent prevalence rates of gonorrhoea, chlamydia, trichomonas, and syphilis

\begin{tabular}{|c|c|c|c|c|c|c|c|c|c|}
\hline & \multicolumn{3}{|c|}{ Pregnant women } & \multicolumn{3}{|c|}{ Symptomatic women } & \multicolumn{3}{|c|}{ Female commercial sex workers } \\
\hline & $\begin{array}{l}\text { Comores } \\
(n=3160)\end{array}$ & $\begin{array}{l}\text { Mauritius } \\
(n=3309)\end{array}$ & $\begin{array}{l}\text { Seychelles } \\
(n=3240)\end{array}$ & $\begin{array}{l}\text { Comores } \\
(n=393)\end{array}$ & Mauritius & $\begin{array}{l}\text { Seychelles } \\
(n=3170)\end{array}$ & $\begin{array}{l}\text { Comores } \\
(n=398)\end{array}$ & $\begin{array}{l}\text { Mauritius } \\
(\mathrm{n}=3104)\end{array}$ & Seychelles \\
\hline Gonorrhoea & 0 & 0 & 1.3 & 5.8 & - & 1.8 & 5.7 & 4.0 & - \\
\hline Chlamydia & 10 & 15 & 6.7 & 15 & - & 6.5 & 10.3 & 18.0 & - \\
\hline Trichomonas & 6.2 & 1.3 & 0.4 & 6.5 & - & 6.5 & - & 14.0 & - \\
\hline Syphilis & 5.0 & 0 & 2.1 & 4.4 & - & 2.4 & 11.3 & 32.0 & - \\
\hline
\end{tabular}


S - Sensitive (or positive for $\beta$-Lactamase) $\square$ IR - Intermediate/resistant (or negative for $\beta$-Lactamase)

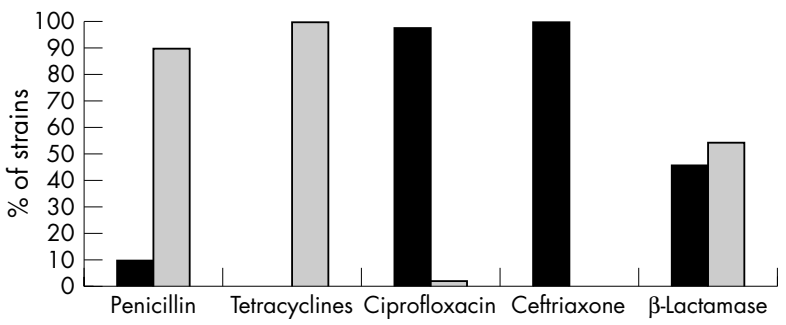

Figure 2 Per cent of drug resistance in 52 isolates of Neisseria gonorrhoeae, Mauritius, 1997/98.

Seychelles. ${ }^{11-13}$ The cumulative prevalence over the total population was $0.08 / 1000,0.15 / 1000$, and $1.0 / 1000$ in the three countries, respectively. The peak in the annual incidence of new cases was reached in 1995 in the Comoros and Seychelles, and in 1997 in Mauritius. The 10 year mean positivity rate of HIV tests among low risk populations (pregnant women and blood donors) was 0.07 in the Seychelles (20 982 tests); the trend was not on the increase (0.05 in 1997). In the Comoros, one single positive test was obtained in these populations over 10 years (26 739 tests). Among hospital inpatients the 10 year mean positivity rates were 0.2 and 0.06 in Seychelles and the Comoros, on a slightly increasing trend (1.1 and 0.3 in 1997). HIV seroprevalence among female prostitutes (a sample of about 100 women) in the Comoros was 0 in 1994 and $0.9 \%$ in 1998; in Mauritius, it was 6.7\% in 1998.

\section{NATURE OF THE SOCIAL AND SEXUAL NETWORKS}

There are important differences in the social structure in the three countries. In the Seychelles the majority of the population is Christian, and a great deal of intermarriage has blurred racial differences, with $68 \%$ being predominantly black African in origin. In Mauritius ethnicity is even more diverse and comprises Asians (51\%, the majority of Indian origin), mixed European and African origin (31\%), and SinoMauritians. The most diffuse religion is Hinduism, with a minority of Christians and Muslims. Religious and cultural attitudes inhibit discussion of sexuality in the home, in schools, or in the media. The Comorian society is much more homogeneous, based on traditional values and deeply rooted in the Islamic culture. Social classes, hierarchy, and networking are built within this framework for the vast majority of the rural Comorian population. Women are marginalised from social and political decision making.

The Comoros, Mauritius, and Seychelles are so small that a distinction between urban and rural environment is of little significance, and movements of populations within the country (internal migration, local labourers) do not play a role in spreading diseases.

Commercial sex is illegal in the three countries. Prostitution does occur in the Comoros and Mauritius, but it is not reported from the Seychelles, despite the presence of a very important tourism industry. Prostitution in the Comoros occurs in bars, small hotels, and popular quarters of the urban environment. It is mainly directed towards local clients. In a prospective questionnaire study almost all the women knew the way of spreading of HIV, but only $43 \%$ knew about the protective effect of the male condom, and $28 \%$ used it regularly. ${ }^{14}$ In Mauritius, there are prostitutes who offer sexual services to visiting foreigners. The existence and the importance of women offering services to both foreigners and local people has not been investigated so far. Alcoholism and drug addition seem to be a problem in a minority of the population in Mauritius only.

\section{DISCUSSION}

The hypothesis that STIs evolve in response to control programmes can be tested if a careful description of the STI epidemiology is available. This prerequisite is not met in the Indian Ocean region, and many other resource-poor countries are likely to share similar difficulties owing to the general weakness of the reporting systems for communicable diseases. Neither long term nor short term perspectives are available because case notification systems are still based on the aetiological diagnosis of diseases (including syphilis, gonorrhoea, chancroid, and trichomoniasis), while the capacity to make an aetiological diagnosis is poor in the capitals and non-existent elsewhere. A feasible target for future development of the epidemiological information system in these countries is represented by the definition of trends of STI syndromes. A shift from an aetiological to a syndromic recording system has been accepted in principle but it will take some time and persistent political commitment before it is implemented. Therefore prospective longitudinal data may not be available for years.

However, the scanty and scattered nature of the available information does suggest that STIs are in a hyperendemic, non-concentrated phase: high rates in the general population in the Comoros (that is, phase II of the Wasserheit model), with somewhat lower rates in the Seychelles, and possibly Mauritius (phase $\mathrm{II}^{\prime}$ ), reflecting a different socioeconomic pattern and efficiency of control programmes.

The seeming paradox in STI prevalence in low and high risk populations when comparing the Comoros with Seychelles and Mauritius deserves comment. Available data show the highest prevalence rates in the general population in the Comoros, while the prevalence among female sex workers is the lowest. This is likely to reflect a different epidemic phase and different transmission patterns in the three countries: the STI epidemics seem to be less concentrated and less core dependent in the Comoros. However, these speculations are based on a very limited amount of information, and deeper epidemiological insight is warranted to exclude biases in the data.

The highest STI rates in the Comoros are likely to be caused by the combined effect of the unfavourable economic situation (with poor general health indicators) and the weakness of STI control efforts. A measure of the latter factor is provided by the comparison of the drug resistance pattern of gonococcal isolates with treatment guidelines for gonorrhoea. In 1998, while the chemosensitivity pattern showed high resistance rates for penicillin and tetracyclines (fig 2), ampicillin, doxycycline, and ciprofloxacine were the recommended first line drugs in the Comoros, Mauritius, and Seychelles, respectively. In that situation STI patients with gonorrhoea were unlikely to be cured when reporting to public health services in the Comoros and Mauritius. In the latter country, however, the private health system may have provided more efficient alternative sources of care.

Seychelles notify a larger number of STI cases but this is likely to reflect a higher efficiency of the reporting system, as the prevalence of STIs in the general population is actually the lowest in the region. In particular, chlamydia rates are lower than in the neighbouring countries, possibly because Seychelles is the only one where significant contact tracing activities are carried out. The reduction of the endemicity of Chlamydia trachomatis infection requires not only an efficient public health system but also specific control interventions. ${ }^{15}$

Mauritius is likely to be in an intermediate epidemiological situation: STI rates seem to be low in the general population but the presence of a much larger diversity in the socioeconomic structure of the society may allow for the emergence of sexual networks of high transmitters. The quality of the health care system is also diverse: public services are generally weak, but more efficient care may be obtained in the private sector. 
Differences in accessibility to efficient care services may thus determine a greater "core dependency" of the STI epidemics. The very high rates of STI among female commercial sex workers are alarming. In addition to socioeconomic conditions and the efficiency of the public health system, it is likely that STI rates in commercial sex workers are the results of the degree of risk behaviours and the magnitude and diversity of client networks.

HIV prevalence is very low in the region, and the epidemic appears to be at an earlier phase in the Comoros than in Seychelles and Mauritius. This observation is consistent with the hypothesis that STIs do not concentrate in core groups in the Comoros. The reasons for the low prevalence of HIV infection in the area are not well understood, as major social and health factors prevailing in the neighbouring sub-Saharan Africa are present in the region as well. One possible protective factor in the Indian Ocean appears to be the limited existence of links between local networks (social and sexual) and external networks, or the scarcity of bridge populations. In theory, newly introduced STIs are expected not to give rise to epidemics unless they enter core groups. ${ }^{16}$ A study on the species distribution of candida isolates from vaginal samples in the Comoros found a relatively low ratio of Calbicans to non-albicans species. ${ }^{17}$ Among the latter group, $C$ sake constituted $28 \%$ of all isolates. Because this species has been reported very rarely from Europe and other industrialised countries, this study supports the hypothesis of a limited linkage between sexual networks within and outside the Comoros. If this hypothesis is correct the increasing prevalence rates among commercial sex workers in Mauritius might represent a particularly dreadful early sign of the impending epidemic in that country.

The concept that identification of the phase of the epidemic is important in defining appropriate control interventions may be of limited value where STI prevalence is still high and gen-

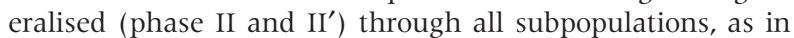
most developing countries. It has been proposed that in a phase II situation initiation of STI-preventive efforts simultaneously in subpopulations in which spread networks are located and in those in which maintenance networks are located might well result in earlier, more rapid, and more sustained declines in STD morbidity. ${ }^{1}$ This concept is easy to accept but difficult to apply. It may be argued that the scarcity of available resources in such a setting should not be directed towards defining the phase, but rather to identifying sustainable ways of implementing old preventive interventions or discovering and implementing new ones.

The importance of the understanding of social and sexual networks dynamics as an alternative approach and a key component in the design of STI control interventions proposed by Wasserheit and $\mathrm{Aral}^{1}$ might have great potential even for populations with phase I and II STI epidemics. Current reporting systems in the Indian Ocean region do not allow for the measurements of concentrations of the epidemics in terms of sexual preference (men who have sex with men), social problems (drug abuse), or even gender and age. Therefore we know little, if anything, about the characteristics in terms of density, concurrency, permeability, and mixing patterns of sexual and social networks and their impact on the spread of STI in the region. The understanding of network dynamics might actually represent one of the "new bullets" we are looking for to exploit the scarce available resources for STI control in a rational way. This may already be a crucial step in the identification of target core groups for focused interventions in Mauritius and the Seychelles, where the epidemics move towards concentrated groups.

Controlling symptomatic STI is an effective tool for reducing the efficiency of HIV transmission. ${ }^{18}$ The Indian Ocean provides a unique opportunity to curb the HIV epidemic in its nascent phase through the strengthening of STI control programmes. Making effective and appropriate health services available should be regarded as the first priority for STI control in the region and, whenever possible, core groups should be identified and targeted to interrupt transmission within such networks. Routinely collected STI surveillance, based on syndromes and coupled with special studies, would still be essential to identify priority intervention areas and monitor trends. Novel efforts to understand social and sexual network dynamics specific to the region should be included in the list of research and surveillance priorities.

\section{ACKNOWLEDGEMENT}

This project received financial support under the contract 7 ACP RPR 28 of the European Commission.

\section{Authors' affiliations}

A Matteelli, C Pizzocolo, S Caligaris, F Castelli, G Carosi, Clinic of Infectious and Tropical Diseases, University of Brescia, Brescia, Italy H Abdou Bakar, M Toyb, AIDS and STD Division, Ministry of Health, the Comoros

N Pyndiah, Laboratory of Virology and STD, Ministry of Health, Mauritius

N E Udonwa, A G Gedeon, Primary Health Care Directorate, Ministry of Health, Seychelles

C Farina, Institute of Microbiology, Ospedali Riuniti, Bergamo, Italy

\section{REFERENCES}

1 Wasserheit JN, Aral SO. The dynamic topology of sexually transmitted disease epidemics: implication for preventive strategy. J Infect Dis 1996; 174(suppl 2):S201-13.

2 Blanchard JF, Moses S, Greenawa C, et al. The evolving epidemiology of chlamydia and gonococcal infections in response to control programme in Winnipeg, Canada. Am J Public Health 1998:88: 1496-502.

3 The World Bank. World development record 1993: investing in health. New York: Oxford University press, 1993

4 Gerbase AC, Rowley JT, Mertens TE. Global epidemiology of sexually transmitted diseases. Lancet 1998;351(suppl III):2-4.

5 Josse R, Dioumoi A, Ouledi A, et al. Nouvelle approche sanitarie en République Fédérale Islamique des Comores. Med Trop 1995;55:205-8

6 Toyb M, Lombart JP, Farina C, et al. Prevalence of STD pathogens among pregnant women in Moroni, Comores. Abidjan, Côte d'Ivoire: Xth International Conference on AIDS and STD in Africa, December 1997.

7 Carosi G, Toyb M, Lombart JP, et al. Prevalence of STD pathogens among female STD patients in Moroni, Comores. Abidjan, Côte d'Ivoire: Xth International Conference on AIDS and STD in Africa, December 1997.

8 Carosi G, Brewer R, Francoise M, et al. Syndrome at presentation and etiologic agents among female STD patients in the Seychelles. Abidjan, Côte d'Ivoire: Xth International Conference on AIDS and STD in Africa, December 1997.

9 Udonwa NE, Matteelli A, Nwosu J, et al. Sensitivity and specificity of a risk score method for the detection of cervical infection in female STD patients with vaginal discharge in the Seychelles. Abidjan, Côte d'lvoire: Xth International Conference on AIDS and STD in Africa, December 1997.

10 Nwosu J, Udonwa NE, Farina C, et al. Syndrome at presentation and etiologic agents among male STD patients in the Seychelles. Abidjan, Côte d'Ivoire: Xth International Conference on AIDS and STD in Africa, December 1997.

11 Toyb M, Lombart JP, Binti Abdou A, et al. SIDA et infection a VIH en le République Fédérale Islamique des Comores (1988-1995). Med Trop 1997;57:59-61.

12 Pyndiah MN, Jowaheer B, Perumal J, et al. Evolution du VIH à Maurice. Bull Soc Pathol Exot 1998; 91: 67

13 Carosi G, Pyndiah NH, Abdou Barar H, et al. Notified AIDS and HIV infection cases in the Indian Ocean Region, 1987-1997. Geneva: Xth World AIDS Conference, June 1998.

14 Abdou Bacar H. Prévention contre les MST et SIDA entre les protituées. Geneva: Xth World AIDS Conference, June 1998.

15 Mertz KJ, Levine WC, Mosure DJ, et al. Trends in the prevalence of chlamydial infections: the impact of community wide testing. Am J Public Health 1997; 24:169-75.

16 May RM, Anderson RM. Transmission dynamics of HIV infection. Nature 1987;326: 137-42

17 Farina C, Malighetti V, Lompart JP, et al. Yeasts from vaginal exudates in the Comoros Islands. J Mycol Med 2000;10:91-3.

18 Grosskurt H, Mosha F, Todd J, et al. Impact of improved treatment of STD on HIV infection in rural Tanzania: randomized controlled trial. Lancet 1995;350:530-6. 